Psychological Medicine

cambridge.org/psm

\section{Correspondence}

Cite this article: Fujitake E, Chen KJP (2020). Letter to Editor: Maternal depression and inflammation during pregnancy. Psychological Medicine 50, 2460-2461. https://doi.org/ $10.1017 /$ S0033291719003398

Received: 29 August 2019

Accepted: 4 November 2019

First published online: 4 December 2019

Author for correspondence:

Kieran Jian Peng Chen,

E-mail: Jian.chen14@imperial.ac.uk

\title{
Letter to Editor: Maternal depression and inflammation during pregnancy
}

Eri Fujitake and Kieran Jian Peng Chen (D)

Faculty of Medicine, Imperial College London, London, UK

Dear Editor,

Lahti-Pulkkinen et al. raises interesting points on how depressive symptoms during pregnancy, or a diagnosis of depression prior to pregnancy, may be associated with a pro-inflammatory state (Lahti-Pulkkinen et al., 2019). They also found that pre-pregnancy obesity has a significant interaction with this relationship, potentiating the body's inflammatory response. The association between inflammatory cytokines and adverse pregnancy outcomes, such as preterm labour, has been well established (Goldenberg, Hauth, \& Andrews, 2000), highlighting the importance of this study. The strengths of the study, including the large sample size and the multiple blood samples taken throughout gestation, provide valuable information to the field of maternal mental health.

The authors used high sensitivity CRP (hsCRP) and glycoprotein acetyl as surrogate markers for the low-grade inflammation seen in depression. Depression is associated with an elevated CRP in the general population, however, it is unknown whether in pregnancy, these low levels are significant enough to generate a pathological outcome (Kashanian, Aghbali, \& Mahali, 2013; Valkanova, Ebmeier, \& Allan, 2013). There is still much debate regarding the role of inflammation in complications of pregnancy such as pre-eclampsia and intrauterine growth restriction. For example, preeclampsia may directly lead to oxidative stress, leading to inflammation and raised CRP (Sánchez-Aranguren et al., 2014). On the other hand, a chronic level of inflammation itself may lead to altered placental formation thus predisposing to pre-eclampsia (Ferguson et al., 2017). There is also a complex bidirectional relationship between maternal mental health and physiological well-being. Inflammatory biomarkers can be affected by patient factors including gestational weight gain, metabolic syndrome and antidepressant use; therefore inflammatory markers must be interpreted with caution (Howren, Lamkin, \& Suls, 2009; Pearson et al., 2003).

The study does not comment on maternal and neonatal outcomes due to the nature of their methodology. Therefore, we cannot observe the possible effects of increased CRP or glycoprotein acetyl on adverse outcomes of pregnancy. Further studies, including postpartum follow-up may be necessary to determine a causal relationship. It may also be interesting to investigate the effect of depression on inflammatory markers whose roles have already been implicated in pathways affecting preterm labour, such as IL-6 and PGE2 (Sykes et al., 2012). Albeit more invasive, samples of biomarkers could also be taken directly from cervical mucus or vaginal fluid (Sawada et al., 2006), which may provide a superior representation of how maternal psychological health affects inflammatory markers within the reproductive environment.

We agree with the authors' conclusion that antenatal depression or depression prior to pregnancy may lead to low grade inflammation. It is well understood that depression may be an independent risk factor for adverse outcomes during pregnancy (Alder et al., 2007). This paper provides good insight into a possible mechanism of why antenatal depression may lead to complications during pregnancy and affect foetal well-being. However, more work in this area is required to underpin a causal relationship to understand the extent to which maternal mental health may lead to adverse outcomes.

Conflict of interest. None.

\section{References}

Alder, J., Fink, N., Bitzer, J., Hösli, I., \& Holzgreve, W. (2007). Depression and anxiety during pregnancy: a risk factor for obstetric, fetal and neonatal outcome? A critical review of the literature. The Journal of Maternal-Fetal \& Neonatal Medicine, 20(3), 189-209.

Ferguson, K. K., Meeker, J. D., McElrath, T. F., Mukherjee, B., \& Cantonwine, D. E. (2017). Repeated measures of inflammation and oxidative stress biomarkers in preeclamptic and normotensive pregnancies. American Journal of Obstetrics and Gynecology, 216(5), 527.e1-527.e9.

Goldenberg, R., Hauth, J., \& Andrews, W. (2000). Intrauterine infection and preterm delivery. The New England Journal of Medicine, 342, 35233-37333. Retrieved from http://www.nejm.org (Accessed 29 August 2019).

Howren, M. B., Lamkin, D. M., \& Suls, J. (2009). Associations of depression with C-reactive protein, IL-1, and IL-6: a meta-analysis. Psychosomatic Medicine, 71(2), 171-186.

Kashanian, M., Aghbali, F., \& Mahali, N. (2013). Evaluation of the diagnostic value of the first-trimester maternal serum high-sensitivity C-reactive protein level for prediction of pre-eclampsia. Journal of Obstetrics and Gynaecology Research, 39(12), 1549-1554. doi: 10.1111/jog.12105. 
Lahti-Pulkkinen, M., Girchenko, P., Robinson, R., Lehto, S. M., Toffol, E., Heinonen, K., ... Räikkönen, K. (2019). Maternal depression and inflammation during pregnancy. Psychological Medicine, 1-13. doi: 10.1017/ S0033291719001909.

Pearson, T. A., Mensah, G. A., Alexander, R. W., Anderson, J. L., Canon, R. O., 3rd, Criqui, M., ... Vinicor, F. (2003). Markers of inflammation and cardiovascular disease. Circulation, 107(3), 499-511. doi: 10.1161/ 01.CIR.0000052939.59093.45.

Sánchez-Aranguren, L. C., Prada, C. E., Riaño-Medina, C. E., \& Lopez, M. (2014). Endothelial dysfunction and preeclampsia: role of oxidative stress. Frontiers in Physiology, 5, 372. doi: 10.3389/fphys.2014.00372.
Sawada, M., Otsuki, K., Mitsukawa, K., Yakuwa, K., Nagatsuka, M., \& Okai, T. (2006). Cervical inflammatory cytokines and other markers in the cervical mucus of pregnant women with lower genital tract infection. International Journal of Gynecology \& Obstetrics, 92(2), 117-121. doi: 10.1016/ j.ijgo.2005.10.004.

Sykes, L., MacIntyre, D. A., Yap, X. J., Teoh, T. G., \& Bennett, P. R. (2012). The Th1:Th2 dichotomy of pregnancy and preterm labour. Mediators of Inflammation, 2012, 1-12. doi: 10.1155/2012/967629.

Valkanova, V., Ebmeier, K. P., \& Allan, C. L. (2013). CRP, IL-6 and depression: a systematic review and meta-analysis of longitudinal studies. Journal of Affective Disorders, 150(3), 736-744. doi: 10.1016/J.JAD.2013.06.004. 